\title{
A Comprehensive Reference Source for the Researchers Involved in Image Enhancement Field - A Review
}

\author{
Emrah Irmak \\ Department of Biomedical Engineering, Karabuk University, Karabuk, Turkey \\ Email address: \\ emrahirmak@karabuk.edu.tr \\ To cite this article: \\ Emrah Irmak. A Comprehensive Reference Source for the Researchers Involved in Image Enhancement Field - A Review. International \\ Journal of Psychological and Brain Sciences. Vol. 2, No. 5, 2017, pp. 109-119. doi: 10.11648/j.ijpbs.20170205.12
}

Received: October 14, 2017; Accepted: October 25, 2017; Published: December 3, 2017

\begin{abstract}
Image enhancement is the processing of a given image so that the result is more suitable than the original image for a particular profession for future automated image processing, such as analysis, detection, segmentation and recognition. The essential target of image enhancement is to minimize noise from a digital image by keeping the intrinsic information of the image preserved. The main difficulty in image enhancement is determining the criteria for enhancement therefore; more than one image enhancement techniques are empirical and require interactive procedures to obtain satisfactory results. In this paper robust image enhancement algorithms are discussed, implemented to noisy images and compared according to their robustness. The algorithms are especially able to improve the contrast of medical images, fingerprint images and selenography images by means of software techniques. When deciding that one image has better quality than another image, quality measure metrics are needed. Otherwise comparing image quality just by visual appearance may not be objective because images could vary from person to person. That is why quantitative metrics are crucial to compare images for their qualities. In this paper Peak Signal to Noise Ratio (PSNR) and Mean Squared Error (MSE) quality measure metrics are used to compare the image enhancement methods systematically. All the methods are validated by the performance measures with PSNR and MSE. It is believed that this paper will provide comprehensive reference source for the researchers involved in image enhancement field.
\end{abstract}

Keywords: Image Enhancement Algorithm, Histogram Matching, Histogram Equalization, Fuzzy Set Theory, Quality Measure Metrics

\section{Introduction}

\subsection{Motivation, Concept and Importance}

Image enhancement is a powerful tool which is widely used in computer vision. It is a popular sub-specialization of image processing. Image enhancement is the improvement of image quality to a better and more understandable level for visual appearance for future automated image processing, such as analysis, detection, segmentation and recognition [1]. Improving interpretability or perception of data in images can be thought as main goal of image enhancement process so that human viewers or future automated image processing can benefit from the new enhanced image. [2]. When dealing with image enhancement it must be kept in mind that image enhancement implementation doesn't have effect upon the intrinsic information content of the data. What is effect is to heighten the dynamic range of the intensity values of image [3].
Image enhancement techniques can be divided into two main groups;

1. Spatial Domain Methods operate directly on pixels. The most significant advantage of spatial domain technique is that they are quite simple to understand and are not very complex which is ideal for real time implementations. The main disadvantage of spatial domain methods is that they are not robust enough for sustainability. Fuzzy logic image enhancement and histogram equalization methods can be included into this group. The operation can be formulated as;

$$
g(x, y)=T[f(x, y)]
$$

For this equation $\mathrm{g}, f$ and $T$ stands for output image, input image and operation on $f$, respectively.

2. Frequency Domain Methods operate on frequency components which is Fourier transform of the image [4]. Frequency domain methods use mathematical functions or signals such as Fourier transform, discrete wavelet transform, 
and discrete cosine transform, which deals with frequency. After that they operate directly on the transform coefficients of the image by manipulating the transform coefficients. The most significant advantage of frequency domain method is that the computation is very simple and complexity is very low. The main disadvantage of frequency methods is that they are local operated. Hence, they cannot simultaneously enhance all parts of image very well and it is also difficult to automate the image enhancement procedure. The operation can be formulated as;

$$
g(x, y)=f(x, y) * h(x, y)
$$

For this equation $\mathrm{g}, f$ and $h$ stands for output image, input image and transfer function, respectively [5].

Frequency domain methods involve 3 basic steps,

1. Transform the input image into its Fourier transform
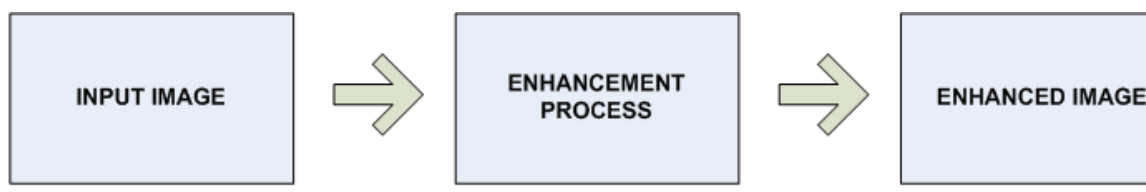

2. Apply the transfer function

3. Inverse Fourier transform is applied to get enhanced image

The type of the noise removing algorithm is directly related to the type of the noise corrupting the image. Additive noise (Gaussian and Impulse noise) and multiplicative noise (Speckle noise) are mainly encountered noise types in the images. Figure 1 is considered as basic and simple representation of image enhancement processes. To begin with, input image which is to be enhanced is taken into digital form environment. On the other hand, judgement about choosing proper image enhancement algorithm is made Last but not least, image is processed using selected image enhancement technique and lastly improved image by image enhancement is displayed as output.

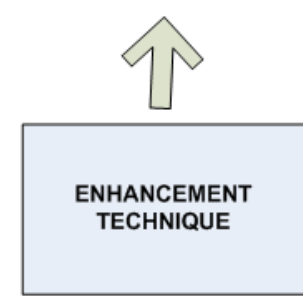

Figure 1. Image Enhancement Algorithm.

\subsection{Literature Survey}

Over the years a variety of image enhancement methods have been proposed with the developments in image processing field. These methods have been independently studied for several different applications, resulting in a large body of research. Although there is a wide range of methodologies, classification and comparison of techniques become difficult because each technique is often designed for specific applications and not necessarily for specific types of problems or data. However the approaches differ in information on which enhancement relies. Briefly stated, the researchers or scientists who intend to enhance images should establish the connection between the changes in the images/targets of enhancement and the type of enhancement methods which can most appropriately be applied.

The work done in literature by various researchers can be summarized as follows;

K. M. Yasmin et. al. [6] studied on brain image enhancement techniques. Brain images contain anatomic sense for neurologic research, diagnosis and treatment. Therefore to evaluate brain images becomes a crucial issue. In that paper brain image enhancement techniques were compared to analyze the brain images precisely in order to effectively diagnose and examine the diseases and problems.

J. K. Hasikin et. al. [7] made use of fuzzy set theory for their image enhancement purposes. This is a low contrast image enhancement technique. This technique is worked by maximizing fuzzy measures contained in the image. Image is enhanced by modification of membership function.

R. Arun et. al. [8] suggested a special Hybrid Technique by using both gray level transformation algorithm and power law transform together to enhance the contrast of the image. This technique has ability of enhancing various kinds of images. What is more, this technique has control over the contrast of the image to the desired degree. is by definition straightforward in theory and practice.

Agaian et. al. [9] proposed logarithmic transform domain histogram and histogram equalization for image enhancement This paper helps choose the best parameters and transform for each enhancement. A number of experimental results are presented to illustrate the performance of the proposed algorithms.

Wadud et. al. [10] studied an a smart contrast enhancement technique based on conventional histogram equalization (HE) algorithm.

L. Xiaoying et. al. [11] proposed image fusion for image enhancement applications. Several different evaluation methods and fusion strategies were discussed and compared.

Mohan et. al. [12] presented a useful survey on enhancement of magnetic resonance images by specifying noise characteristics of MR images.

Image quality measure metrics are also crucial in an 
enhancement point of view. Therefore there are a variety of quality measure metrics in literature. Chow et. al. [13] reviewed medical image, such as magnetic resonance, computed tomography and ultrasound, quality measure metrics.

Researcher interested in more specific aspects of image enhancement can refer to publications by Kamran et. al. for ultrasound image enhancement [14], Suganthi et. al. for edge enhancement [15], Anand et. al. for mammographic image enhancement [16], Hossain et. al. for contrast enhancement [17], Babu et. al. for speckle reduction using fuzzy logic on coefficient of variations [18], Deka et. al. for removal of correlated speckle noise [19], Janani et. al. for infrared image enhancement techniques [20], Imtiaz et. al. for endoscopic image enhancement [21], Bhattacharya et. al. for localized image enhancement [22]. Table 1 shows a useful and comprehensive comparison of recently published papers in image enhancement field.

Table 1. Comparison of the Latest Published Papers in Image Enhancement Field.

\begin{tabular}{|c|c|c|c|}
\hline Author & Year & Operating Domain & Model \\
\hline M. Yasmin [6] & 2012 & Transform domain, spatial domain & Noise reduction, resolution, segmentation, noise suppression \\
\hline J. K. Hasikin [7] & 2012 & Spatial domain & Fuzzy-based contrast modification \\
\hline R. Arun $[8]$ & 2011 & Spatial domain & Alpha rooting based hybrid Procedure \\
\hline S. S. Agaian [9] & 2007 & Transform domain, spatial domain & $\begin{array}{l}\text { Transform coefficient histogram-based image enhancement } \\
\text { algorithms }\end{array}$ \\
\hline M. A. Wadud [10] & 2007 & Spatial domain & Conventional histogram equalization \\
\hline L. Xiaoying [11] & 2011 & Transform domain, spatial domain & Image fusion method evaluation on sharpness \\
\hline L. S. Chow [13] & 2016 & Spatial domain & Subjective assessments, subjective assessments \\
\hline K. Binaee [14] & 2014 & Filtering & Speckle reduction \\
\hline S. S. Suganthi [15] & 2014 & Filtering & Edge enhancement for segmentation \\
\hline S. Anand [16] & 2013 & Transform domain & Directionlet transform (DT) \\
\hline M. B. Hossain [17] & 2014 & Spatial domain & $\begin{array}{l}\text { Multipurpose Beta Optimized Recursive Bi-Histogram } \\
\text { Equaliza-tion (MBORBHE) }\end{array}$ \\
\hline J. J. J. Babu [18] & 2016 & Filtering & Smoothening of the edges \\
\hline M. S. Imtiaz [21] & 2013 & Spatial domain & Image enhancement at gray level and color reproduction \\
\hline Saumik Bhattacharya [22] & 2014 & Spatial domain & Singular value decomposition (SVD) approach \\
\hline
\end{tabular}

Table 1. Continued.

\begin{tabular}{|c|c|c|c|}
\hline Author & Year & Processing Techniques & Application \\
\hline M. Yasmin [6] & 2012 & Non-linear and non-iterative noise reduction method. & Brain images \\
\hline J. K. Hasikin [7] & 2012 & Contrast enhancement, grayscale enhancement & Grayscale images \\
\hline R. Arun $[8]$ & 2011 & Alpha rooting technique & A variety images \\
\hline S. S. Agaian [9] & 2007 & $\begin{array}{l}\text { The logarithmic transform domain histogram and } \\
\text { histogram equalization }\end{array}$ & A human visual system-based \\
\hline M. A. Wadud [10] & 2007 & Contrast enhancement & Brain image, synthetic image, natural image \\
\hline L. Xiaoying [11] & 2011 & Improvement the perception of information & The cameraman image, gray an color images \\
\hline L. S. Chow [13] & 2016 & Medical image quality assessments & MR, CT, ultrasound images \\
\hline K. Binaee [14] & 2014 & Fuzzy rule based filter & Ultrasound images \\
\hline S. S. Suganthi [15] & 2014 & non-linear isotropic diffusion filter & Grayscale breast thermal image \\
\hline S. Anand [16] & 2013 & Sharpening Technique (ST) & Mammographic X-ray images \\
\hline M. B. Hossain [17] & 2014 & A new contrast enhancing method & Ultrasound images \\
\hline J. J. J. Babu [18] & 2016 & An adaptive fuzzy logic approach & Ultrasound images \\
\hline B. Deka [19] & 2013 & De-speckling algorithm & Photographic images \\
\hline V. Janani [20] & 2014 & A variety enhancement techniques & Infrared images \\
\hline Saumik Bhattacharya [22] & 2014 & Contrast enhancement & Localized image enhancement \\
\hline
\end{tabular}

This paper is divided into four sections, namely: Introduction to image enhancement field (Section 1), Image enhancement methods (Section 2), Experimental results (Section 3), Conclusion and future work (Section 4).

\section{Methodology}

Many image enhancement algorithms have been developed to improve the appearance of images. In this paper four commonly used image enhancement techniques are explained, compared and corresponding experimental results are shown. These enhancement techniques are as following:

I. Fuzzy Set Theory Image Enhancement Method

II. Histogram Equalization Image Enhancement Method

III.Histogram Matching Image Enhancement Method

IV.Equalized Histogram Equalization Image
Enhancement Method

\subsection{Fuzzy Set Theory Image Enhancement Method}

The issue whether a pixel should become darker or 
brighter than it already is, can be considered as the principal goal of the fuzzy set theory image enhancement technique [23] Figure 2 can be considered as the key point algorithm of the fuzzy set theory image enhancement algorithm. To begin with input image is taken into digital form environment. In addition to that image histogram is computed to be able to decide whether corresponding image histogram is applicable for fuzzy set theory with s-shape membership function image enhancement method or not. Last but not least it is concluded that fuzzy logic s-shape image enhancement method is applicable to this specific noisy image if histogram range lies down in a narrow band. On top of that processed image is converted to fuzzy plane. At the following step membership function is modified by means of appropriate membership function modification formula. Lastly enhanced image is displayed as output.

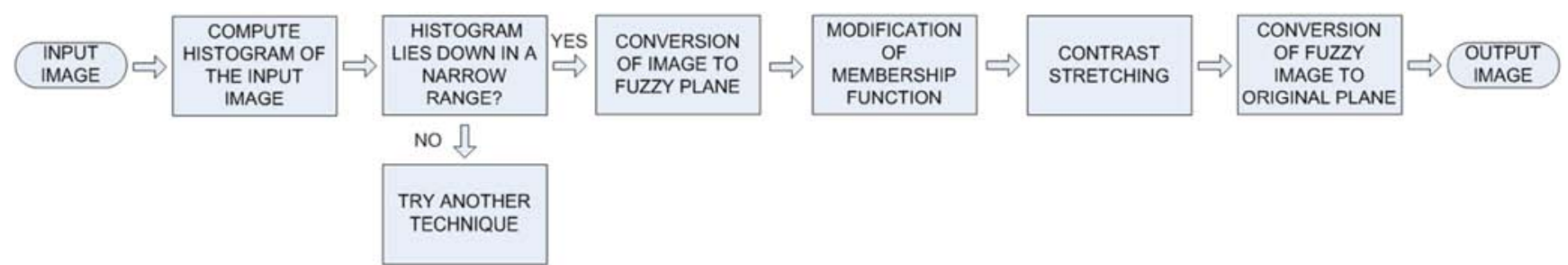

Figure 2. Common flowchart for Fuzzy Set Theory Enhancement

\section{Algorithm:}

Step-1: Find image histogram using any software technique. If histogram range lies down in a narrow band fuzzy set theory with s-shape membership function image enhancement method is applicable to this specific noisy image.

Step-2: Once decided that aforementioned method is applicable assign minimum intensity value $\left(g_{\min }\right)$ and maximum intensity value $\left(g_{\max }\right)$ of the image.

Step-3: Next is defuzzification step. Shift minimum intensity value to 0 , maximum intensity value to 1 , and other intensity values between 0 and 1 using fuzzy formula shown below [24].

Conversion to Fuzzy Plane:

$$
\mu_{(g)}=\frac{g-g_{\min }}{g_{\max }-g_{\min }}
$$

Step-4: This step involves membership function modification. There are many membership functions in literature. Decision for selecting membership function is dependent on image intrinsic content such as image histogram. In this paper s-shape membership function is used now that image histogram is suitable. Membership function is modified using following formula.

S-Shape Membership Function:

$$
S(z ; a, b, c)=\left\{\begin{array}{cc}
0 & \mathrm{z}<\mathrm{a} \\
2\left(\frac{\mathrm{z}-\mathrm{a}}{\mathrm{c}-\mathrm{a}}\right)^{2} & \mathrm{a} \leq \mathrm{z} \leq \mathrm{b} \\
1-2\left(\frac{\mathrm{z}-\mathrm{c}}{\mathrm{c}-\mathrm{a}}\right)^{2} & \mathrm{~b}<\mathrm{z} \leq \mathrm{c} \\
1 & \mathrm{z}>\mathrm{c}
\end{array}\right.
$$

Step-5: To stretch contrast of the processed image multiply each pixel by a constant number.

Step-6: Convert image to its original plane from fuzzy plane (defuzzification) and examine the enhanced image [25].

\subsection{Histogram Equalization Image Enhancement Method}

Histogram equalization is a popular method in low level image enhancement using the histogram of the image. The principle is that an image is said to be the best in visual appearance, when its histogram looks like the uniform distribution. Probability density function is the key point operator in the histogram equalization method. A new image with equally distributed intensity level from the lowest pixel value $(0)$ to the highest pixel value $(L-1)$ is formed using probability density function. By means of this idea, handicapped intensity values are increased whereas excessive intensity values are decreased therefore the contrast of the image is increased.

Algorithm:

Step-1: Compute probability density function (pdf) of image $x$. The probability of an occurrence of a pixel of level $i$ in the image is:

$$
p d f(i)=\frac{\text { number of the pixel with intensity } i}{\text { total number of pixels in image } x} 0<i<L
$$

In fact $p d f(i)$ is histogram of the image that is normalized to $[0,1] . L$ is the total number of gray levels in the image, which is generally 256 .

Step-2: Calculate cumulative distribution function $(c d f)$ considering $(p d f)$ for each pixel.

$$
c d f(i)=\sum_{k=0}^{L-1} p d f(i)
$$

The aim is to create a transformation of the form $y=T(x)$ to produce a new image $\mathrm{y}$, with a flat histogram. Such an image would have a linearized cumulative distribution function (cdf) across the value range.

Step-3: Get the value of the pixels by multiplying $c d f(i)$ by $L-1$ and then round it to the nearest integer.

$$
s_{k}=(\mathrm{L}-1) \sum_{k=0}^{L-1} \operatorname{pdf}(i)
$$

\subsection{Histogram Matching Image Enhancement Method}

Histogram matching is an extensive revision of the histogram equalization. Difference is that in histogram equalization, the histogram of the output image is desired to be uniformly distributed whereas in histogram matching 
method, the histogram of the output image is manipulated as to follow according to that users determine. It becomes the best approach to have the control of adjusting the shape of the histogram. The method used to generate a processed image that has a specified histogram is called histogram matching [26].

Algorithm:

Step-1: Compute the probability density function of the input image, $p\left(r_{j}\right)$.

$$
p\left(r_{j}\right)=\frac{n_{j}}{n}
$$

where

$n_{j}=$ the number of the pixels with gray level $r_{j}$.

$n=$ the total number of the pixels in the image.

Step-2: Evaluate the transformation function, $T(r)$.

$$
T\left(r_{k}\right)=\sum_{j=0}^{k} p\left(r_{j}\right) \quad k=0,1,2,3, \ldots, L-1
$$

where

$p\left(r_{j}\right)=$ probability density function of image pixel intensity values.

This transformation function is actually the cumulative distribution function.

Step-3: Find transformation function $G_{z}$.

$$
G\left(z_{k}\right)=\sum_{i=0}^{k} p\left(z_{i}\right) i=k=0,1,2,3, \ldots, L-1
$$

$p\left(z_{i}\right)=$ specified probability density function which the output image is desired to have. $p\left(z_{i}\right)$ is known.

Step-4: Obtain the inverse transformation function $G^{-1}$.

Step-5: Obtain the output image by applying Equation 11 to all the pixels to input image. The result of this procedure is an image whose gray levels $z$, have the specified probability density function.

$$
z_{k}=G^{-1}\left(T\left(r_{k}\right)\right) k=0,1,2,3, \ldots, L-1
$$

\subsection{Equalized Histogram Equalization Image Enhancement Method}

Equalized histogram equalization method is an improved conventional histogram equalization method. First of all the input image is enhanced using the algorithm described in section 2.2. Obtained output image is summed up with input image. By this way loss parts in output image can be recovered [27], [28].

Algorithm:

Step-1: Compute probability density function $(p d f)$ of the input image.

$$
p d f(i)=\frac{\text { number of the pixel with intensity } i}{\text { total number of pixels in image } x} 0<i<L
$$

Step-2: Calculate cumulative distribution function (cdf) considering $p d f(i)$ for each pixel.

$$
c d f(i)=\sum_{k=0}^{L-1} p d f(i)
$$

Step-3: Get the value of the pixels by multiplying $c d f(i)$ by $L-1$ and then round it to the nearest integer.

$$
s_{k}=\sum_{k=0}^{L-1}(L-1) * p d f(i)
$$

Step-3: Add new image to the input image to get the enhanced output image.

$$
E=s_{k}+x
$$

\begin{tabular}{|c|c|c|c|c|c|}
\hline Enhancement Technique & Instrumentality & Operating Domain & Application Field & Complexity & Processing Speed \\
\hline FUZZY & Histogram & Spatial & Specific & Relatively Complex & Relatively Slow \\
\hline $\mathrm{HE}$ & Histogram & Spatial & Wide-range & Simple & Fast \\
\hline HS & Histogram & Spatial & Wide-range & Simple & Fast \\
\hline HS & Histogram & Spatial & Wide-range & Simple & Fast \\
\hline EHE & Histogram & Spatial & Intermediate & Intermediate & Relatively Fast \\
\hline
\end{tabular}

where

$$
\begin{aligned}
& E=\text { enhanced output image } \\
& x=\text { input image }
\end{aligned}
$$

After presenting each method in details Table 2 is a compact notation of FUZZY, HE, HS and EHE. Before proceeding to experimental results it is a good idea to take a look at the table comparison below.

Table 2. Comparison Chart of Image Enhancement Techniques in Question.

\section{Experimental Results}

Above image enhancement methods are implemented to three different image types, these image types are Medical Image, Fingerprint Image and Selenography Image. Results are demonstrated in Figure 3, Figure 4 and Figure 5, respectively. Corresponding image histograms are also shown in these figures. Figure $3 \mathrm{~A}$ is a noisy MR image of a patient's knee before enhancement process. Figure $3 \mathrm{~B}$ is enhanced MR image using Equalized Histogram Equalization Image Enhancement Method (EHE), Figure 3C is enhanced MR image using Histogram Matching Image Enhancement
Method (HS), Figure 3D is enhanced MR image using Histogram Equalization Image Enhancement Method (HE) and lastly Figure 3E is enhanced MR image using Fuzzy Set Theory Image Enhancement Method (Fuzzy). Figure 4 and Figure 5 are results for Fingerprint and Selenography Images, respectively. An image is said to be the best in visual appearance, when its histogram looks like the uniform distribution [29]. From the figures it can be seen that image histograms are uniformed after enhancement process comparing with histograms before enhancement process. In image processing it is quite difficult to compare the enhancement results by visual appearance and just looking at the images with naked eye [30]. Therefore to compare the 
result and to make comment image quality metrics are used in this paper.

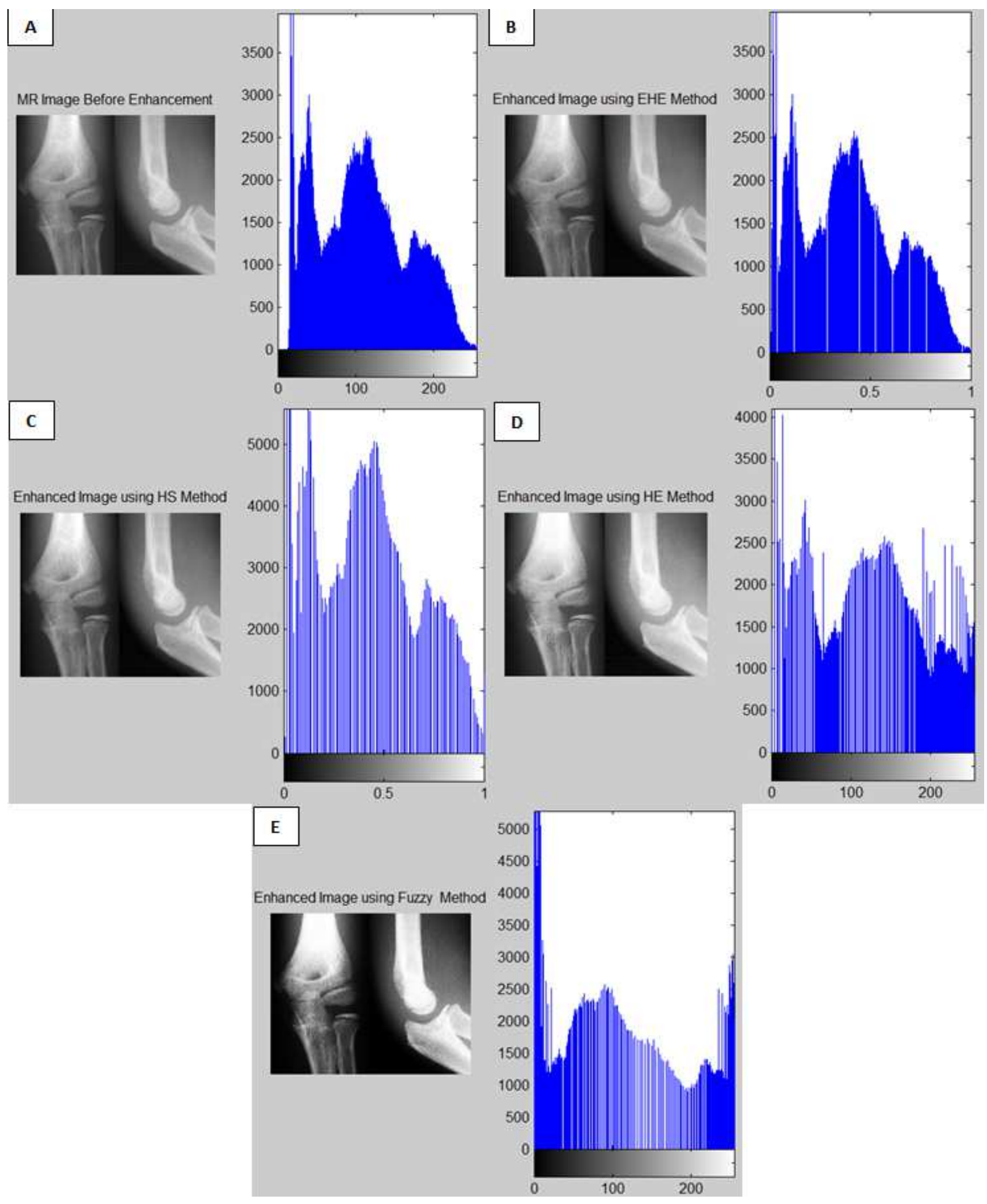

Figure 3. (A) Noisy MR image, enhancement results and corresponding histograms using method (B) EHE, (C) HS, (D) HE and (E) Fuzzy. 


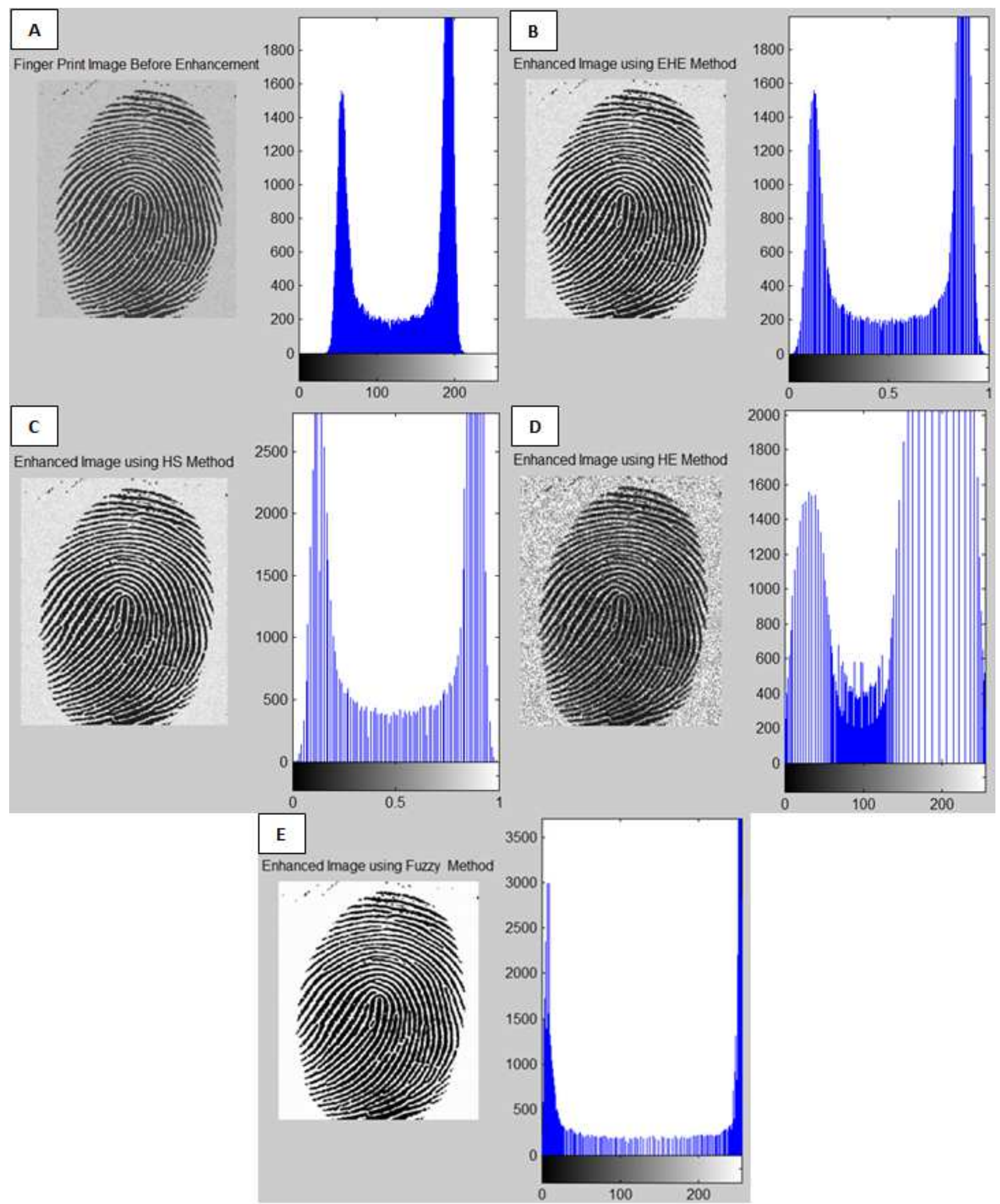

Figure 4. (A) Noisy fingerprint image, enhancement results and corresponding histograms using method (B) EHE, (C) HS, (D) HE and (E) Fuzzy. 


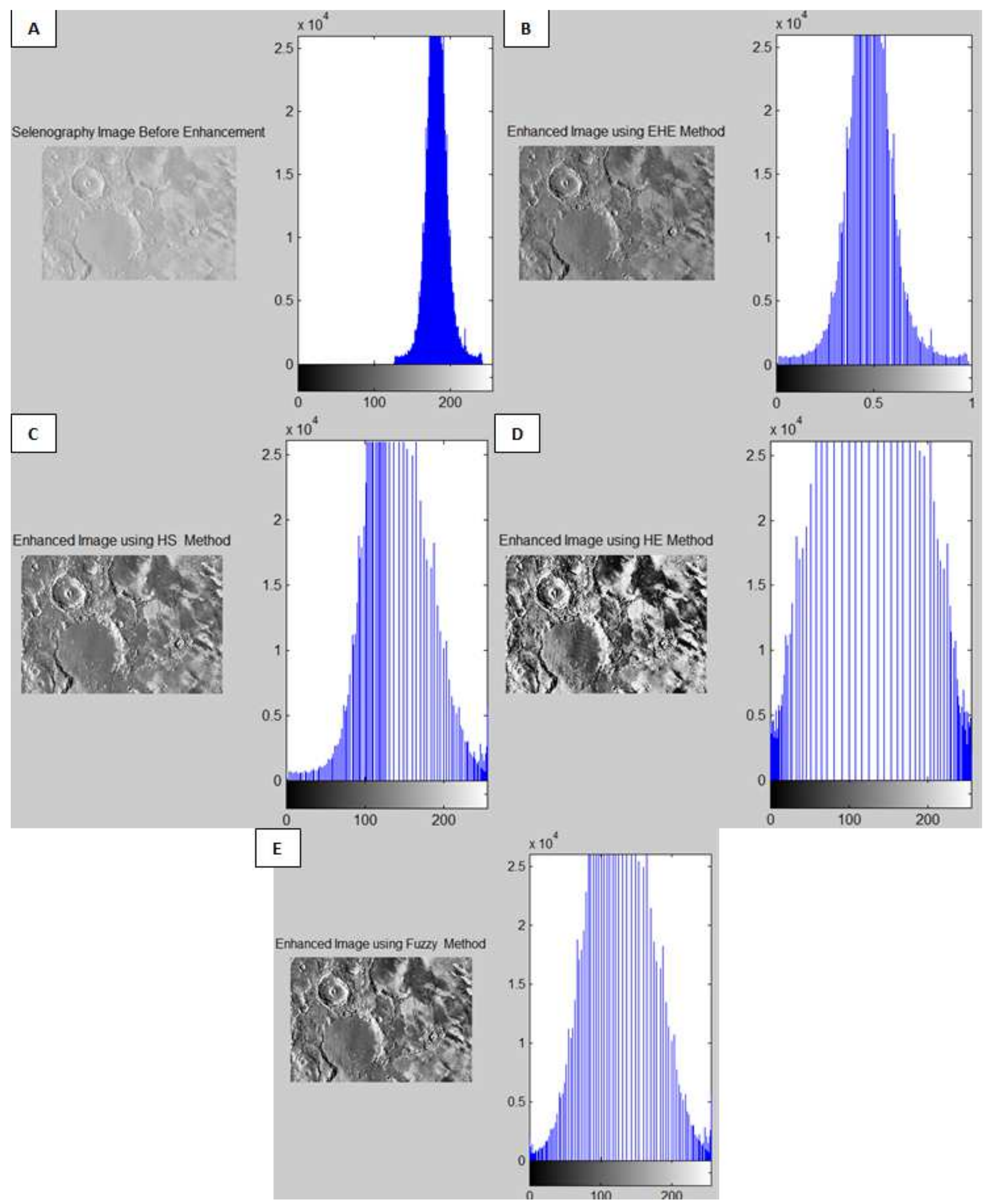

Figure 5. (A) Noisy selenography image, enhancement results and corresponding histograms using method (B) EHE, (C) HS, (D) HE and (E) Fuzzy.

To validate the enhancement in images and the visual quality of image, Peak Signal to Noise Ratio (PSNR) and Mean Squared Error (MSE) are often used to compare the results of methods. When deciding that one image has better quality than another image, quality measure metrics are needed [31]. Otherwise comparing image quality just by visual appearance may not be objective because images could vary from person to person [32]. That is why quantitative 
metrics are necessary to compare images for their qualities [31], [33]. In this paper PSNR and MSE quality measure metrics are used to compare the image enhancement methods systematically. PSNR is the ratio between the maximum possible value (power) of a signal and power of the noise corrupted by the signal.

The golden rule is that the higher the PSNR, the better noisy images has been enhanced and the better the enhancement algorithm. This would occur when we minimize the MSE between the images with respect the maximum signal value of the image.

The mathematical representation of the PSNR is as follows:

$$
P S N R=20 * \log _{10} \frac{M A X_{f}}{\sqrt{M S E}}
$$

where

$f=$ matrix data of our enhanced image

$M A X_{f}=$ maximum signal value that exists in our "enhanced" image

$M S E=$ mean squared error

In image processing the MSE measures the average of the squares of the "errors", that is, the difference between the enhanced image and noisy image. MSE is a risk function, corresponding to the expected value of the squared error loss or quadratic loss [34]. The less the MSE the best result for enhancement [35].

The mathematical representation of the MSE is as follows [36], [37]:

$$
M S E=\frac{1}{m * n} \sum_{0}^{m-1} \sum_{0}^{n-1}\|f(i, j)-g(i, j)\|^{2}
$$

where

$g=$ matrix data of our noisy image in question

$m=$ numbers of rows of pixels of the images and $\mathrm{i}$ represents the index of that row

$n=$ number of columns of pixels of the image and $j$ represents the index of that column

In Table 3 the results of the PSNR and MSE for four image enhancement methods are shown with respect to three different image types. For MR image PSNR value of EHE method is 31.7524 . PSNR value for HS method is 30.3380 for HE method is 19.9431 and for Fuzzy method is 17.9600 . As mentioned before the higher the PSNR, the better noisy images has been enhanced and the better the enhancement algorithm [38], [39]. This means that EHE method is best method and Fuzzy method is worst method out of four methods. However fuzzy method is a good enhancement method for low contrast images only. HS and HE method are the other better method respectively. For fingerprint and selenography images similar results are shown in Table 3.

Again looking at Table 3 for MR image it is seen that MSE value for EHE method is 43.4347, for HS method is 60.1563, for HE method is 658.8181 and for Fuzzy method is 1040.1654. It is know that the less the MSE the better result for enhancement [40]. This confirms that the best method is EHE method whereas the worst method is Fuzzy method. HS method and HE method are the other better method respectively. For fingerprint and selenography images similar results are shown in Table 3.

Table 3. Image Quality Measures according to method used.

\begin{tabular}{llll}
\hline IMAGE TYPE & METHOD & PSNR & MSE \\
\hline \multirow{4}{*}{ MR IMAGE } & EHE & 31.7524 & 43.4347 \\
& HS & 30.3380 & 60.1563 \\
& HE & 19.9431 & 658.8181 \\
& FUZZY & 17.9600 & 1040.1654 \\
FINGER PRINT & EHE & 19.6638 & 702.5886 \\
IMAGE & HS & 19.1625 & 788.5485 \\
& HE & 18.5780 & 902.1604 \\
& FUZZY & 13.4963 & 2907.1000 \\
SELENOGRAPHY & EHE & 12.2846 & 3842.6000 \\
IMAGE & HS & 11.1830 & 4952.0000 \\
& HE & 10.7374 & 5487.1000 \\
& FUZZY & 10.1107 & 6338.8000 \\
\hline
\end{tabular}

\section{Conclusion}

Image enhancement is an important sub-area of digital image processing field. More than one image enhancement techniques occur in image processing. These image enhancement techniques improve visual appearance of any portion or feature of the images by suppressing the information in other portions or features. Image enhancement techniques propose a wide variety of algorithms for improving images to obtain visually acceptable images. The selection of such techniques is a matter of the specific task and image content. In this paper four commonly used and useful image enhancement methods are analyzed for their effectiveness. For enhancement purposes three important image types are used as database. These image types are medical image, fingerprint image and selenography image. Four image enhancement methods are implemented to these noisy images and compared according to their robustness with respect PSNR and MSE image quality measure metrics. The algorithms are especially able to improve the contrast of medical images, selenography images and finger prints images by means of software techniques. The results are shown in Table 3. From the table it is said that EHE method is the best method for image enhancement problems. HS method, HE method and Fuzzy method are the other better image enhancement methods respectively. However fuzzy method is a good enhancement method for low contrast images only. This order is validated by using PSNR and MSE. PSNR and MSE results show the same result deduction and confirm each other.

\section{References}

[1] M. M. H. Chowdhury, M. E. Islam, N. Begum, and M. A. A. Bhuiyan, "Digital image enhancement with fuzzy rule-based filtering," 10th Int. Conf. Comput. Inf. Technol., pp. 1-3, 2007.

[2] P. Journal, I. Society, and R. Sensing, "Theme oriented enhancement of sea surface temperature in thermal," vol. 15, no. 2,1987 . 
[3] E. Irmak, K. Ileri, and A. Ozkahraman, "Concept and Implementation of Fuzzy Set Theory Technique for Image Enhancement Purposes," no. 1, pp. 1-4.

[4] K. Venkateshwarlu and M. Bala, "Image enhancement using fuzzy inference system," Comput. Sci. Eng., vol. 60, no. 16, pp. 8-13, 2010.

[5] G. X. Jiu, J. F. Jiao, and L. Xiang, "Image enhancement method based on fuzzy set and subdivision," $20113 \mathrm{rd}$ Int. Conf. Aware. Sci. Technol., pp. 174-176, 2011.

[6] M. Yasmin, M. Sharif, and S. Masood, "Brain image enhancement-A survey," World Appl. Sci, vol. 17, no. 9, pp. 1192-1204, 2012.

[7] K. Hasikin and N. A. M. Isa, "Enhancement of the Low Contrast Image Using Fuzzy Set Theory," 2012 UKSim 14th Int. Conf. Comput. Model. Simul., no. March, pp. 371-376, 2012.

[8] R. Arun, M. Nair, R. Vrinthavani, and R. Tatavarti, "An Alpha Rooting Based Hybrid Technique for Image Enhancement," Image (IN)., no. August, 2011.

[9] S. S. Agaian, B. Silver, and K. A. Panetta, "Transform coefficient histogram-based image enhancement algorithms using contrast entropy," IEEE Trans. Image Process., vol. 16, no. 3, pp. 741-758, 2007.

[10] M. Abdullah-Al-Wadud, M. Kabir, M. Akber Dewan, and O. Chae, "A Dynamic Histogram Equalization for Image Contrast Enhancement," IEEE Trans. Consum. Electron., vol. 53, no. 2, pp. 593-600, 2007.

[11] X. Fang, J. Liu, W. Gu, and Y. Tang, "A method to improve the image enhancement result based on image fusion," 2011 Int. Conf. Multimed. Technol., pp. 55-58, 2011.

[12] J. Mohan, V. Krishnaveni, and Y. Guo, "A survey on the magnetic resonance image denoising methods," Biomed. Signal Process. Control, vol. 9, no. 1, pp. 56-69, 2014.

[13] L. S. Chow and R. Paramesran, "Review of medical image quality assessment," Biomed. Signal Process. Control, vol. 27, pp. 145-154, 2016.

[14] K. Binaee and R. P. R. Hasanzadeh, "An ultrasound image enhancement method using local gradient based fuzzy similarity," Biomed. Signal Process. Control, vol. 13, no. 1, pp. 89-101, 2014.

[15] S. S. Suganthi and S. Ramakrishnan, "Biomedical Signal Processing and Control Anisotropic diffusion filter based edge enhancement for segmentation of breast thermogram using level sets," Biomed. Signal Process. Control, vol. 10, pp. 128136, 2014.

[16] S. Anand, R. S. S. Kumari, S. Jeeva, and T. Thivya, "Directionlet transform based sharpening and enhancement of mammographic X-ray images," Biomed. Signal Process. Control, vol. 8, no. 4, pp. 391-399, 2013.

[17] M. B. Hossain, K. W. Lai, B. Pingguan-Murphy, Y. C. Hum, M. I. Mohd Salim, and Y. M. Liew, "Contrast enhancement of ultrasound imaging of the knee joint cartilage for early detection of knee osteoarthritis," Biomed. Signal Process. Control, vol. 13, no. 1, pp. 157-167, 2014.

[18] J. Jai Jaganath Babu and G. Florence Sudha, "Adaptive speckle reduction in ultrasound images using fuzzy logic on
Coefficient of Variation," Biomed. Signal Process. Control, vol. 23, pp. 93-103, 2016.

[19] B. Deka and P. K. Bora, "Removal of correlated speckle noise using sparse and overcomplete representations," Biomed. Signal Process. Control, vol. 8, no. 6, pp. 520-533, 2013.

[20] V. Janani, "Infrared Image Enhancement Techniques - A Review,” Int. Conf. Curr. trend Eng. Technol., 2014.

[21] M. S. Imtiaz, T. H. Khan, and K. Wahid, "New Color Image Enhancement Method for Endoscopic Images," no. Icaee, pp. 19-21, 2013.

[22] S. Gupta, V. K. Subramanian"Localized image enhancement" @ iitk. ac. in, E. E Dept., IIT Kanpur,” Ieee, 2014.

[23] S. H. Contreras Ortiz, T. Chiu, and M. D. Fox, "Ultrasound image enhancement: A review," Biomed. Signal Process. Control, vol. 7, no. 5, pp. 419-428, 2012.

[24] P. P. S. J, P. K. Rajeswari, and I. M. April, "Membership Function modification for Image Enhancement using fuzzy logic," vol. 2, no. 2, pp. 114-118, 2013.

[25] C. Reshmalakshmi and M. Sasikumar, "Image Contrast Enhancement using Fuzzy Technique,” no. 1, pp. 861-865, 2013.

[26] "DIP_2E_Digital Image Processing 2nd Gonzales Woods.pdf.".

[27] C. Vol, "Equalize The Histogram Equalization for Image enhancement," vol. 1, no. 5, pp. 14-21, 2012.

[28] E. Irmak, E. Erçelebi, and A. H. Ertaş, "Brain tumor detection using monomodal intensity based medical image registration and MATLAB," Turkish J. Electr. Eng. Comput. Sci., vol. 24, pp. 2730-2746, 2016.

[29] M. F. Al-Samaraie, "A New Enhancement Approach for Enhancing Image of Digital Cameras by Changing the Contrast," Int. J. Adv. Sci. Technol., vol. 32, pp. 13-22, 2011.

[30] C. Chaudhary and M. K. Patil, "Review of Image Enhancement Techniques Using Histogram," Int. J. Appl. or Innov. Eng. Manag., vol. 2, no. 5, pp. 343-349, 2013.

[31] P. Mohammadi, "Subjective and Objective Quality Assessment of Image: A Survey," arXiv Prepr. arXiv, no. June, pp. 1-50, 2014.

[32] R. L. Easton and Jr, "Fundamentals of Digital Image Processing," no. November, 2010.

[33] H. R. Wu and K. R. Rao, Digital Video Image Quality and Perceptual Coding. 2005.

[34] H. R. Sheikh and A. C. Bovik, "Image information and visual quality.," IEEE Trans. Image Process., vol. 15, no. 2, pp. $430-444,2006$.

[35] H. L. Tan, Z. Li, Y. H. Tan, S. Rahardja, and C. Yeo, "A perceptually relevant mse-based image quality metric," IEEE Trans. Image Process., vol. 22, no. 11, pp. 4447-4459, 2013.

[36] P. Kaushik and Y. Sharma, "Comparison of different image enhancement techniques based upon Psnr \& Mse," Int. J. Appl. Eng. Res., vol. 7, no. 11 SUPPL., pp. 2010-2014, 2012.

[37] C. S. Varnan, A. Jagan, J. Kaur, D. Jyoti, and D. S. Rao, "Image Quality Assessment Techniques in Spatial," Int. J. Comput. Sci. Technol., vol. 2, no. 3, pp. 177-184, 2011. 
[38] N. Thakur and S. Devi, "A New Method for Color Image Quality Assessment," Int. J. Comput. Appl., vol. 15, no. 2, pp. 10-17, 2011.

[39] M. M. Kazi, A. V Mane, R. R. Manza, and K. V Kale, "Comparison of fingerprint enhancement techniques through Mean Square Error and Peak-Signal to Noise Ratio," Int. J.
Comput. Sci. Eng. \{ISSN\} 0975-3397, vol. 3, no. 1, pp. 266$270,2011$.

[40] E. Irmak and A. H. Ertas, "A review of robust image enhancement algorithms and their applications" The 4th IEEE International conference on Smart Energy Grid Engineering, August 21-24, 2016. 